\title{
Infall models of Class 0 protostars
}

\author{
Ray Jayawardhana \\ Department of Astronomy, University of California, Berkeley, CA 94720 \\ Lee Hartmann and Nuria Calvet \\ Harvard-Smithsonian Center for Astrophysics, 60 Garden Street, Cambridge, MA 02138; \\ email: lhartmann@cfa.harvard.edu
}

\begin{abstract}
We have carried out radiative transfer calculations of infalling, dusty envelopes surrounding embedded protostars to understand the observed properties of the recently identified "Class 0" sources. To match the far-infrared peaks in the spectral energy distributions of objects such as the prototype Class 0 source VLA 1623, pure collapse models require mass infall rates $\sim 10^{-4} M_{\odot} \mathrm{yr}^{-1}$. The radial intensity distributions predicted by such infall models are inconsistent with observations of VLA 1623 at sub-mm wavelengths, in agreement with the results of André et al. (1993) who found a density profile of $\rho \propto r^{-1 / 2}$ rather than the expected $\rho \propto r^{-3 / 2}$ gradient. To resolve this conflict, while still invoking infall to produce the outflow source at the center of VLA 1623, we suggest that the observed sub-mm intensity distribution is the sum of two components: an inner infall zone, plus an outer, more nearly constant-density region. This explanation of the observations requires that roughly half the total mass observed within 2000 AU radius of the source lies in a region external to the infall zone. The column densities for this external region are comparable to those found in the larger Oph A cloud within which VLA 1623 is embedded. This decomposition into infall and external regions is not unique, owing to uncertainty in the structure of the molecular gas outside of the infall zone, which in turn implies some uncertainty in estimating the infall rate. Nevertheless, the environment of Oph A is so dense that any protostellar clouds which fragment out are likely to collapse at very high infall rates, consistent with our spectral energy distribution modeling. The extreme environments of Class 0 sources lead us to suggest an alternative or additional interpretation of these objects: rather than simply concluding with André et al. that Class 0 objects only represent the earliest phases of protostellar collapse, and ultimately evolve into older "Class I" protostars, we suggest that many Class 0 sources could be the protostars of very dense regions, and Class I objects found in lower-density regions may be in comparable evolutionary states.
\end{abstract}


Subject headings: ISM: clouds, Radiative transfer, Stars: Formation, Stars: Pre-Main Sequence 


\section{Introduction}

An important aim of the study of young stellar objects (YSOs) has been to classify them along an evolutionary sequence. According to the widely used classification scheme of Lada \& Wilking (1984) and Lada (1987), YSOs are divided into three classes based on the slopes of their infrared spectral energy distribution (SED). The reddest objects, dubbed Class I sources, are thought to be protostars surrounded by infalling envelopes. Their SEDs are consistent with dust emission from envelopes with infall rates near those predicted by the standard theory of protostellar formation, which invokes free-fall collapse from an initially thermally-supported, isothermal, singular molecular cloud core in hydrostatic equilibrium (Adams \& Shu 1986; Adams, Lada \& Shu 1987). Kenyon, Calvet \& Hartmann (1993, hereafter $\mathrm{KCH}$ ), in particular, were able to match the observed SEDs of Class I sources in Taurus-Auriga with rotating infall models of the type described by Terebey, Shu \& Cassen (1984). This agreement between theory and observation is generally seen as testimony to the success of the standard model of protostellar collapse.

Recently, André, Ward-Thompson \& Barsony (1993, hereafter AWB) identified YSOs characterized by blackbody-like SEDs that peak at submillimeter wavelengths. The low dust temperatures $\left(T_{d} \sim 20 \mathrm{~K}\right)$ and large dust masses inferred for these sources led AWB to suggest that these objects constitute a new class of objects, called "Class 0", which might be even younger than typical Class I objects. Observationally, the defining characteristics of Class 0 sources include a high ratio of submillimeter to bolometric luminosity $\left(\mathrm{L}_{\text {submm }}^{\lambda>350 \mu} / \mathrm{L}_{b o l}\right.$ $\left.>5 \times 10^{-3}\right)$, invisibility at near-infrared wavelengths, and the presence of molecular outflows (Barsony 1994). André \& Montmerle (1994) argued that Class 0 sources have more circumstellar mass than stellar mass. In this picture, Class 0 sources have not yet assembled the bulk of their final stellar mass and are still in the main accretion phase. Consequently, the authors argue that Class 0 sources are the youngest protostars, and subsequently evolve into Class I sources (see André, Ward-Thompson, \& Barsony $2000=$ AWB2).

Following AWB's identification of the prototype Class 0 source VLA 1623 in the rich star-forming core $\rho$ Ophiuchi A, several other objects of the class have been found in Orion B (Ward-Thompson et al. 1995), Serpens (Hurt \& Barsony 1996), Cep E (Lefloch et al. 1996), and Perseus (O'Linger et al. 1997) molecular clouds. In a spectroscopic survey of 47 candidate protostars, Mardones et al. (1997) report that Class 0 sources have stronger infall signatures -i.e., larger negative velocity shifts- than Class I sources, and attribute that result to a difference in the physical conditions in the circumstellar envelopes of the two source types.

In order to understand better the observed characteristics of this 'youngest' population of YSOs, we have performed detailed radiative transfer calculations of dust envelope 
emission in Class 0 sources, following the methods described by $\mathrm{KCH}$ and Calvet et al. (1994). We also study the observed radial intensity profiles of the spatially-resolved prototype Class 0 source VLA 1623 at three submillimeter wavelengths. In both cases, we compare the observations to the predictions of the standard model, and explore the possibility that environments exterior to the infall region can affect the spectral energy distribution and the inferred density gradients.

\section{Standard Model}

\subsection{Methods and Parameters}

Following KCH, we adopt the Terebey, Shu \& Cassen (1984, hereafter TSC) solution for a uniformly rotating protostellar cloud with a radial density distribution initially approaching that of a singular isothermal sphere, $\rho(r) \propto r^{-2}$. However, unlike TSC, we assume an outer boundary to the envelope, $r_{\text {out }}$. Inside the collapse region, the infall velocity approaches the free fall value, $v \propto r^{-1 / 2}$, and the density distribution converges toward $\rho \propto r^{-3 / 2}$, because the mass infall rate, $\dot{M}_{i}$ is constant. Inside the centrifugal radius, $R_{c}$, material falls onto a circumstellar disk rather than radially onto the central object.

We use the angle-averaged density distribution to construct the spherically symmetric source function, $S_{\nu}=\sigma_{\nu} J_{\nu}+\left(1-\sigma_{\nu}\right) B_{\nu}$, where $\sigma_{\nu}$ is the albedo and $B_{\nu}$ is the Planck function. Scattering increases the flux at short wavelengths, $\lambda \lesssim 3 \mu \mathrm{m}$. We then derive the emergent flux from the envelope using $S_{\nu}$ for the source function and the angle-dependent density, $\rho(r, \theta)$, for the opacity.

The angular momentum of the infalling material modifies the density distribution from the purely-radial infall solution at $r \lesssim R_{c}$. However, for the models considered here, the observable emission originates from envelope radii much larger than typical disk sizes $\sim 100$ $\mathrm{AU}$, and so the value of $R_{c}$ is unimportant. Similarly, we do not include envelope cavities

such as those produced by bipolar outflows. $\mathrm{KCH}$ pointed out that envelope cavities were needed to explain the near-infrared emission of some Taurus Class I sources; but the very large optical depths characteristic of the models considered here prevent the escape of any significant amount of near-infrared scattered light from the envelope.

Thus, our models consist of a central stellar source, with bolometric luminosity $L$, surrounded by an envelope that is essentially spherically-symmetric, with inner radius $r_{i n}$ and outer radius $r_{\text {out }}$. The inner radius of the envelope is set by the dust destruction radius, which is assumed to occur at $T \sim 1500 \mathrm{~K}$. For $r_{\text {out }}$, we adopt values roughly equivalent to the observed sizes of the sources being modeled. The models have then three free parameters: 
the source luminosity $L, r_{\text {out }}$, and the density of the envelope (see below). We used Draine $\&$ Lee (1984) opacities for $\lambda \leq 200 \mu m$ and a shallower $\beta=1.5$ (where $\kappa_{\nu} \propto \nu^{\beta}$ ) opacity law for longer wavelengths.

\subsection{Model Results}

\subsubsection{Spectral energy distribution}

We began by constructing a grid of models with a wide range of envelope densities for source luminosities consistent with the observed values for Class 0 sources of interest. These models were defined in terms of $\rho_{1}$, the density at $r=1 \mathrm{AU}$ in the limit that $R_{c} \rightarrow 0$. The mass infall rate is related to this density parameter and the central mass by

$$
\dot{M}=1.9 \times 10^{-6} M_{\odot} \mathrm{yr}^{-1}\left(\frac{\rho_{1}}{10^{-14} \mathrm{~g} \mathrm{~cm}^{-3}}\right)\left(\frac{M_{*}}{1 M_{\odot}}\right)^{1 / 2}
$$

The envelope temperature distribution is not very sensitive to the SED of the central source because its radiation is immediately absorbed by the innermost (i.e., large optical depth) regions of the envelope and reradiated at longer wavelengths.

These models show general agreement with the analytic approximation of $\mathrm{KCH}$ for the peak wavelength of the SED:

$$
\lambda_{m} \approx 60\left(\frac{L}{10^{33} \mathrm{erg} \mathrm{s}^{-1}}\right)^{-1 / 12}\left(\frac{\rho_{1}}{10^{-13} \mathrm{~g} \mathrm{~cm}^{-3}}\right)^{1 / 3} \mu \mathrm{m} .
$$

For typical source luminosities and expected central masses, in order to shift the peak of the SED to $\lambda \sim 200 \mu m$, as is observed in Class 0 sources, the models require infall rates $\geq 10^{-4} M_{\odot} y r^{-1}$.

Unfortunately, SEDs of most known Class 0 sources are not well defined observationally. Many of them have been observed only at a couple of sub-millimeter wavelengths, and the IRAS upper limits often do not provide strong constraints in the mid- and far-infrared. Therefore, we restrict our detailed modeling to two sources with relatively well-defined SEDs:

VLA 1623 Located in the $\rho$ Oph A molecular cloud, VLA 1623 is a centrally-peaked source of millimeter emission (André et al. 1990b), a weak $6 \mathrm{~cm}$ radio source (Leous et al. 1991), and the driving source of a strong CO bipolar outflow (André et al. 1990a). The highly collimated outflow has a mechanical luminosity of $L_{\text {flow }} \sim 0.5-2 L_{\odot}$ and a short dynamical timescale $t_{D} \sim 3000-6000$ yrs, making it one of the youngest known flows. 
VLA 1623 is undetected at near- and mid-infrared wavelengths to very low limits. Based on these properties as well as its remarkably cold temperature $(T \sim 20 \mathrm{~K})$, high internal obscuration, apparently massive circumstellar structure and high $L_{\text {submm }} / L_{b o l}$ ratio, AWB proposed it as the prototype of the newly-defined Class 0 sources. Observations with the JCMT-CSO interferometer show an unresolved central source -presumably a circumstellar disk- in VLA 1623. However, its contribution to the total flux at $1.3 \mathrm{~mm}$ and $800 \mu \mathrm{m}$ is $\leq$ 10\% (Pudritz et al. 1996).

In our infall model for VLA 1623, we set $L=1 L_{\odot}$ and $r_{\text {out }}=2000 \mathrm{AU}$, which corresponds to 12 " at the distance of Ophiuchus and is roughly the observed source size in the sub-millimeter. (We adopt $d=160 \mathrm{pc}$ to Oph, the same as AWB.) For the model that best fits the observed SED of VLA 1623 (Fig. 1a), the mass infall rate is $\sim 2 \times 10^{-4} M_{\odot \mathrm{yr}^{-1}}$ for a central stellar mass of $0.5 M_{\odot}$. A lower infall rate model, with $\dot{M} \sim 10^{-5} M_{\odot} \mathrm{yr}^{-1}$, shown as a dotted line in Fig. 1a, does not match the data. Using the same parameterization of opacity as AWB $-\kappa_{\nu}=0.1 \mathrm{~cm}^{2} \mathrm{~g}^{-1}(\nu / 1000 \mathrm{GHz})^{1.5}$ - we also obtain a total envelope mass of $\sim 0.6 M_{\odot}$ for the best-fit model.

HH24MMS This bright source of millimeter emission near the Herbig-Haro object HH24 in the Orion B molecular cloud at a distance of 460 pc was discovered by Chini et al. (1993). It is not detected in the near- or far-infrared, although IRAS limits are rather poor because of source confusion. VLA observations have revealed a $3.6 \mathrm{~cm}$ source coincident with the millimeter peak (Bontemps, André \& Ward-Thompson 1995). Using JCMT observations at 350, 450, 800 and $1100 \mu \mathrm{m}$, Ward-Thompson et al. (1995) have produced a reasonably good SED for HH24MMS, even though its peak is not tightly constrained due to a lack of shorter wavelength detections.

We modeled HH24MMS with $L=2.5 L_{\odot}$ and $r_{\text {out }}=4000 \mathrm{AU}$, corresponding to an angular cloud diameter of 18 ". Our best fit model requires a mass infall rate of $\sim 3 \times 10^{-4} M_{\odot \mathrm{yr}^{-1}}$, assuming $M_{*}=0.5 M_{\odot}($ Fig. 1b).

\subsubsection{Radial intensity profiles}

Since VLA 1623 is slightly extended at sub-millimeter wavelengths, we attempted to compare our "best-fit" model profiles to the observed azimuthally-averaged radial intensity profiles at $350 \mu \mathrm{m}, 450 \mu \mathrm{m}$ and $800 \mu \mathrm{m}$ of AWB. The model output was convolved with the appropriate beam size at each wavelength and scaled to a distance of $160 \mathrm{pc}$. All the profiles, shown as dotted lines in Figure 2, have been normalized to the peak flux values. As André et al. noted, a modified TSC infall model with $\rho \propto r^{-3 / 2}$ cannot reproduce the 
observed profiles, which are significantly shallower.

In our basic model, the temperature at the outer edge of the infalling cloud is not constrained and in fact drops to $\sim 7 \mathrm{~K}$. Given that external heating is likely to keep the cloud surface at $\sim 20 \mathrm{~K}$, we explored the effect of adopting a minimum temperature in the envelope of $T_{\min }=20 \mathrm{~K}$. The result, shown as dashed lines in Figure 2 , is to produce somewhat shallower intensity distributions, but still not shallow enough to fit the observations well.

Our calculations differ from those of Terebey, Chandler \& André (1993) in that we assume an outer boundary to the envelope. The inability to determine the zero flux level in the observed intensity profiles is an additional source of uncertainty in our fits.

\section{Contribution of exterior regions}

The presence of a compact VLA source and a collimated CO bipolar outflow as well as the centrally peaked submillimeter emission strongly suggest that a central source -i.e., a protostar- has already formed in VLA 1623. Moreover, the presence of a compact $(<70 \mathrm{AU}) \mathrm{mm}$ and submm source consistent with a dusty circumstellar disk (Pudritz et al. 1996) emphasizes the likelihood that substantial infall has already occurred. Thus, one would expect to find an infall region characterized by a density distribution of roughly $\rho \propto r^{-3 / 2}$ (see $\S 4.1$ ). In this context the $\rho \propto r^{-1 / 2}$ distribution inferred by AWB from the radial intensity profiles is surprising. 円

One possibility is that material outside the infall region contributes to the observed intensity profile. As clearly seen in the submillimeter continuum maps of $\rho$ Ophiuchi (AWB; Motte et al. 1998), VLA 1623 is indeed surrounded by -and likely embedded in- larger-scale cloud emission. We have attempted to model the exterior cloud material as a uniformly emitting "slab" at 20K. The resulting radial intensity profiles, which include contributions from the infall zone as well as the exterior region, are shown as solid lines in Figure 2. We find that in order to match the observed intensity distributions at $350 \mu \mathrm{m}, 450 \mu \mathrm{m}$ and $800 \mu \mathrm{m}$, the "slab" needs to have a column density $<N_{H 2}>\sim 6 \times 10^{22} \mathrm{~cm}^{-2}$ or $A_{V} \approx 60$, implying $M_{\text {slab }} \approx 0.3 M_{\odot}$ in the 2000 AU radius region. This compares well with typical column densities of $\approx 10^{23}$ in the larger $\rho$ Oph A cloud derived by Motte et al. (1998).

\footnotetext{
${ }^{1} \mathrm{~A} \rho \propto r^{-1 / 2}$ density distribution corresponds to $\Sigma \propto r^{1 / 2}$ surface density distribution. Thus, for the slowly-varying temperature distributions characteristic of the outer envelope $T \approx r^{-0.4}$, the surface brightness distribution would actually increase with increasing radius without including an outer cutoff radius; this parameter has a crucial effect on the resulting intensity distribution.
} 
Could extinction by this outer region make a Class I source appear as a Class 0? A "slab" of the kind we propose, with $A_{V} \approx 60$, would significantly extinct emission from the inner regions at short wavelengths. However, because the extinction of such an outer region is $A_{\lambda}<1$ at wavelengths $\lambda \gtrsim 30 \mu m$, it would not shift the peak of a Class I SED to $\lambda>100 \mu \mathrm{m}$. Therefore, it is unlikely that a Class I source with a relatively flat $\operatorname{SED}$ in $\lambda$ $\mathrm{F}_{\lambda}$ would appear as a Class 0 simply due to external extinction.

Our best-fit, composite model for the SED of VLA 1623 is shown in Figure 3. Using the same opacities as AWB, this object has a total mass of $0.6 M_{\odot}$, divided roughly equally between the central infall zone and the outer, nearly constant-density region. Our composite model still requires a high infall rate of $\sim 10^{-4} M_{\odot} \mathrm{yr}^{-1}\left(\mathrm{M}_{*} / 0.5 \mathrm{M}_{\odot}\right)^{1 / 2}$, i.e., the necessary infall rate is only reduced by a factor of two by the inclusion of the constant density outer region.

Interestingly, model fits to the 'youngest' (i.e., most embedded) protostars in Chandler \& Richer's (2000) sample imply that the power-law index of the envelope density distribution $p$ (defined by $\rho \propto r^{-p}$ ) is lower than that predicted by the standard collapse model. They suggest that a shallow density profile may be maintained in the outer envelope by magnetic fields and/or turbulence. On the other hand, Hogerheijde \& Sandell (2000) find that the density distribution in envelopes around four embedded YSOs in Taurus are fit equally well by a radial power-law index $p=1.0-2.0$ or by a Shu collapse model.

\section{Discussion}

\subsection{Environment and high infall rates}

In the previous section we showed that models of a collapsing isolated cloud can fit the SEDs of Class 0 source if a high infall rate is adopted, but that the resulting surface brightness distributions are inconsistent with observations, as originally found by AWB. We have extended this result to include the effects of external heating, and still find a discrepancy between theory and observation. The surface brightness distributions can be explained with simple infall models only by including an additional emitting component, which could be material in the near environment of the collapsing cloud.

Infall models generally exhibit surface density distributions $\Sigma \propto r^{-1 / 2}$ (implying $\rho \propto r^{-3 / 2}$ in spherical geometry), whether from spherical or flattened clouds, similarity solutions for collapse or numerical time-dependent calculations, with or without cloud magnetization (e.g., Larson 1969; Penston 1969; Shu 1977; Foster \& Chevalier 1993; Galli \& Shu 1993a,b; Hartmann et al. 1994; Hartmann, Calvet, \& Boss 1996; Li \& Shu 1996). 
In isothermal clouds at the point of collapse, many calculations show density distributions which tend to steepen more towards $r^{-2}$ (e.g., Mouschovias 1991, and references therein; Foster \& Chevalier 1993). These models strongly suggest that the observations of Class 0 sources encompass not only an infall region, but external material not necessarily in collapse.

The question then arises: if the external environment contributes a large fraction of the sub-mm flux, can we in fact say anything about infall rates? A static cloud, with density distribution $\rho \sim r^{-1 / 2}$ as considered by AWB, could, if physically realizable, fit the observations in the absence of any infall. (Note that such a static cloud is inconsistent with the standard model of isothermal clouds, in which a $\rho \propto r^{-2}$ density distribution is expected). It seems likely that some fraction of the sub-mm emission arises from an infall region, because some infall must have occurred to produce the central source, and given that pre-stellar cores appear to be much less centrally condensed than Class 0 sources (Ward-Thompson et al. 1994; André et al. 1996; Ward-Thompson, Motte, \& André 1999; AWB2).

Nevertheless, let us take the extreme limit by supposing that all of the sub-mm emission arises from a static cloud. We may then estimate the rate at which this cloud would collapse. For a cloud mass of $\sim 0.6 M_{\odot}$ concentrated within a radius of $2000 \mathrm{AU}$, the free-fall timescale is $\sim 2 \times 10^{4}$ years. Thus, if this cloud were to collapse, it would exhibit an average infall rate of $\sim 3 \times 10^{-5} M_{\odot} y r^{-1}$. This estimated infall rate is not much smaller than the $\sim 10^{-4} M_{\odot} y r^{-1}$ estimated for the pure infalling envelope model based on the SED fitting. Moreover, if the region that fell in to form the outflow source was originally denser than the outer static region, as seems probable, the infall rate could be higher than this estimate. Thus, one is lead to the same order of magnitude infall rate from consideration of the spatial concentration of the local gas, independent of the fraction of emission currently arising from an external cloud rather than the infall region.

High infall rates are also suggested by observations of outflows. In a survey of nearby embedded YSOs, Bontemps et al. (1996) found that Class 0 sources have unusually powerful outflows. The Class 0 objects in their sample lie an order of magnitude above the well-known correlation between the outflow momentum flux (integrated over time) and bolometric luminosity that holds for Class I sources. Since models of bipolar outflows (Shu et al. 1994, Ferreira \& Pelletier 1995) predict that the mass-loss rate is some fraction of the mass-accretion rate, the stronger outflows in Class 0s also call for significantly higher infall rates than in Class Is. Henriksen, André, \& Bontemps (1997) attempt to derive accretion rates as a function of time for Class 0 sources which are consistent with infall at non-constant rates, again finding very rapid mass accumulation is required to explain 
outflow properties.

In addition, from a spectroscopic survey of 47 candidate protostars, Mardones et al. (1997) report that Class 0 sources have stronger infall signatures -i.e., larger negative velocity shifts- than Class I sources. One possible interpretation of their results is that Class 0s have higher density envelopes than Class Is, again implying higher infall rates in Class 0 objects.

Thus, our high infall rates, which imply that Class 0 sources are a short-lived phenomenon, are consistent with previous conclusions in the literature (e.g., AWB). A comparison of source statistics in Taurus-Auriga and Ophiuchus support this conclusion. With an infall rate of $10^{-4} \mathrm{M}_{\odot} \mathrm{yr}^{-1}$, a $0.5 M_{\odot}$ star would be accreted in $\sim 5000$ years, a timescale consistent with the dynamical lifetime of VLA 1623. André \& Montmerle (1994) and Barsony (1994) estimate a lifetime of $\sim 10^{4}$ yr for Class 0 sources in Oph on statistical grounds. In addition, as there are around 200 known pre-main-sequence objects in Taurus, one would expect $5000 \mathrm{yrs} / 10^{6} \mathrm{yrs} \times 200$ or $\sim 1$ Class 0 source; and Taurus-Auriga contains only two "border-line" Class 0 sources, L1527 and L1551 IRS 5 (Chen et al. 1995). (AWB2 suggest that two Class 0 sources are present in Taurus, L1527 and the extremely low-luminosity source IRAM 04191+1522 [André, Motte, \& Bacmann 1999]).

One problem with the picture of high infall rates for Class 0 sources is that much higher accretion rates onto the central protostar should lead to high accretion luminosities. However, Class 0 objects are not very much more luminous than their Class I counterparts. Henriksen, André, \& Bontemps (1997) and André (1997) have suggested several factors that may reduce the theoretical accretion luminosity: in Class 0 sources, the central stellar mass might be lower, the stellar radius probably larger, and the amount of accretion energy dissipated in the wind is also likely to be larger. Another natural explanation is that material falls onto a disk, instead of falling directly onto the central star. However, the material must still somehow flow from the disk to the star at some point in the protostellar phase to build up the central star. It may be that Class 0 sources also undergo "FU Orionis-type" outbursts once enough material piles up in the disk to make it unstable (Kenyon et al. 1990, 1994).

Note also that an "external region" is not necessarily physically unconnected with the Class 0 source; i.e., this material may eventually collapse onto and be incorporated into the central star(s). Whether this is necessarily the case, however, is not clear. 


\subsection{Class 0 vs. Class I: the evolutionary model vs. initial density}

AWB suggested that the Class 0 sources represent the earliest phase of protostellar collapse, based on the large amount of circumstellar material in the near environment (compared with the likely mass of the central protostellar object). Henriksen, André, \& Bontemps (1997) elucidated a picture which in principle provides a theoretical basis for this evolutionary scheme.

Henriksen et al. pointed out that theoretical models of clouds initially in hydrostatic equilibrium, such as the isothermal Bonnor-Ebert sphere (e.g., Foster \& Chevalier 1993), tend to have flat inner density distributions surrounded by an outer envelope of roughly $\rho \propto r^{-2}$. In addition, Henriksen et al. emphasized the observational evidence for flattened central regions in pre-stellar cores mentioned in $\S 4$. When the initially flat inner regions of such a cloud fall in, high mass infall rates are produced (see also Foster \& Chevalier 1993); material from the outer envelope falls in later at slower rates. This leads to time-dependent infall rates, with the highest rates occuring during the earliest phases of collapse, identified as the Class 0 phase.

This picture, however, does not explain why Class 0 sources tend to be found in dense star forming regions. Moreover, while the general picture of decreasing infall rate with increasing age is quite plausible, it is not clear whether such a Class 0 phase would dominate protostellar infall, because the amount of mass in the flattened inner portions of the initial cloud is generally smaller than the mass in the envelope, implying that most of the protostellar mass must be added during the envelope infall (Class I) phase. Henriksen et al. (1997) show that, for a flat region of a cloud to contain half of the total cloud mass, the $\rho \propto r^{-2}$ envelope can only extend out a factor of 1.6 in radius beyond the flat region essentially, the cloud is almost entirely at constant density, with only a small outer envelope region, inconsistent with observations of typical pre-stellar cores. Henriksen et al. suggest that clouds with steeper edges, $\rho \propto r^{-3}$, could alleviate this problem while maintaining the required large factor $\sim 10$ between Class I and Class 0 lifetimes; but they do not show that the very slow infall implied by these models for the Class I phase is consistent with SED models.

Alternatively, one might suppose that the high environmental densities characterizing the birthsites of Class 0 sources guarantee that envelope masses will be very large on small scales. In other words, the Class 0 sources might be the Class I sources of dense star-forming regions.

As a specific example, consider two protostellar clouds of the same mass but formed in regions of differing average density. The characteristic infall rates will scale roughly as the 
cloud mass divided by the free fall time, which in turn scales roughly as the inverse square root of the mean density; thus, $\dot{M} \propto M /<\rho>^{-1 / 2} \propto<\rho>^{1 / 2} \propto R^{-3 / 2}$, where $R$ is the outer radius of the cloud. (Let us fix the central star mass in order to discuss the difference in infall rates.) If we assume that the Class 0 source has an infall rate of $10^{-4} M_{\odot} y r^{-1}$, and a Class I source has an infall rate of $4 \times 10^{-6} M_{\odot} y r^{-1}$, then if the two protostellar clouds originally had the same mass, the factor of 25 in $\dot{M}$ implies that the Class I source would have to be larger in radius by a factor of 8.5. In other words, the mass within a $2000 \mathrm{AU}$ (12 arcsec at $160 \mathrm{pc}$ ) radius in a Class 0 source should be compared to the mass within a radius of $16,000 \mathrm{AU}(\sim 100 \mathrm{arcsec})$ in a Class I source to properly assess the relative evolutionary states of these objects.

The molecular surveys of Myers and collaborators (e.g., Benson \& Myers 1989; Zhou et al. 1989) have indicated that many cores, such as those in Taurus, have substantial spatial extensions. For instance, even the lowest-mass ammonia cores in the survey of Benson \& Myers (1989) have $0.8-0.6 M_{\odot}$ of material, comparable to that of the Class 0 sources, but distributed within structures of (half-power diameters) $\sim 10,000 \mathrm{AU} \sim 0.05 \mathrm{pc}$ rather than $2000 \mathrm{AU}=0.01 \mathrm{pc}$. In many other cases the ammonia core mass is considerably larger than $1 M_{\odot}$ and spread over larger scales. Whether a substantial portion of the material in a typical Taurus molecular cloud core actually is incorporated into a particular protostellar source is not clear -or whether it makes only one star. However, the same could be said of Class 0 sources. Neglect of the possible importance of extended material could bias the comparison between the two types of sources.

Bontemps et al. (1996) attempted a systematic assessment of Class 0 and Class I source masses from measurements of $1.3 \mathrm{~mm}$ continuum emission. In computing dust masses they adopted a "typical" source diameter of 1 arcmin over which to integrate the emission. In doing so, Bontemps et al. recognized that they might be measuring only the "inner" envelope mass of "isolated" protostars such as those in Taurus. Moreover, if our suggestion of the presence of significant "external" material in VLA 1623 is correct, then Class 0 mass estimates might be biased by the inclusion of mass that may not end up on the protostar, especially if integrated over a radius of $30 \operatorname{arcsec} \sim 4800 \mathrm{AU}$, rather than the $2000 \mathrm{AU}$ radius adopted in the previous sections. Given the large differences in densities observed in star-forming regions, the systematic biases in mass estimates from adopting uniform source sizes can be of an order of magnitude, which could easily affect assessments of the relative evolutionary states of Class 0 and Class I sources. Unfortunately, the observational problem of deciding where a protostellar cloud "stops" is likely to be quite difficult. Yet, without consideration of this potential bias, it seems premature to conclude that all Class 0 sources must be in a radically different phase of evolution than Class I sources. 
In Taurus, the source statistics of Class I sources suggest a lifetime of $1-2 \times 10^{5} \mathrm{yr}$ (Kenyon et al. 1990, 1994). If one adopts the infall rates of $\sim 4 \times 10^{-6} M_{\odot} y r^{-1}$ typically estimated from SED fitting (ALS; KCH), then one would expect that approximately $0.4-0.8 M_{\odot}$ is added to protostars during the Class I phase - which is comparable to the total mass accreted onto the average Taurus $\mathrm{T}$ Tauri star. It is difficult to see why the Class I period is not an important part of the protostellar phase in Taurus, unless somehow infall rates have been overestimated by an order of magnitude. If, on the other hand, Class I sources are the protostars of low-density regions like Taurus, then there is no discrepancy to explain. The two borderline Class 0 objects in Taurus (L1551 IRS 5 and L1527; Chen et al. 1995; or L1527 and IRAM 04191+1522; André et al. 1999) could be among the youngest protostellar sources in Taurus (Ladd et al. 1998); however, they might also be objects formed from particularly dense subregions within Taurus.

To summarize, we agree with AWB and AWB2 that the Class 0 sources are very young in an absolute sense because their envelopes must collapse so rapidly. However, we suggest that the high infall rates of Class 0 sources can result also from the high densities of their initial pre-stellar cloud cores rather than from always being the very first phases of collapse. Whether Class 0 sources are systematically younger than the Class I sources in a relative sense, i.e. have a much smaller fraction of their total mass accumulated into a central protostar, is far from clear given the observational biases and uncertainties in defining envelope limits. Some Class 0 sources may also be young in this relative sense, but it is difficult to support the conclusion that they are all the "youngest protostars".

\subsection{Dense environments and the standard picture}

Infall rates on the order of $10^{-4} M_{\odot} \mathrm{yr}^{-1}$ seem to be needed to account for Class 0 sources. This infall rate is much higher than the typical infall rates $\sim 4 \times 10^{-6} M_{\odot} \mathrm{yr}^{-1}$ for Class I sources in Taurus-Auriga $(\mathrm{KCH})$. In the standard singular isothermal sphere model, the infall rate is $\dot{M} \sim c_{s}^{3} / G$; thus the mean Taurus infall rate requires a sound speed $c_{s} \sim 0.25 \mathrm{~km} \mathrm{~s}^{-1}$ or a gas temperature of $18 \mathrm{~K}$ assuming a mean molecular weight of 2.3, only slightly larger than typical temperatures found in the region. In contrast, to obtain infall rates of $\sim 10^{-4} M_{\odot} \mathrm{yr}^{-1}$ in a cloud supported only by thermal gas pressure would require unrealistically high cloud temperatures of about $150 \mathrm{~K}$. Thus, some other source of support must be involved if the cloud is initially in hydrostatic equilibrium.

These considerations emphasize the importance of the density of the protostellar environment to an understanding of Class 0 sources. The related question is how do such dense regions of material arise? If such regions are in hydrostatic equilibrium, turbulent 
motions undoubtedly play a role in supporting denser clouds (e.g., Lizano \& Shu 1989; Fuller \& Myers 1993). On the other hand, since the sources of turbulence are presumably non-isotropic and inhomogeneously distributed (i.e., winds, magnetic waves excited by moving dense gas clumps, etc.) arranging static situations seems very difficult. In addition, supersonic magnetic turbulence may decay rapidly (Stone, Ostriker, \& Gammie 1998). An alternative view is that dense streams of gas in the ISM, however produced, collide and dissipate energy in a shock (e.g., Ballesteros-Paredes, Vázquez-Semadeni \& Scalo 1999), resulting in dense regions which then undergo gravitational collapse. In such a general picture, there is no obvious reason why densities of protostellar regions need be restricted in any way to levels supportable by thermal gas pressure. In this way protostars with dense envelopes and rapid infall rates could be produced without reference to the usual constraints of thermal support (ALS). The Class 0 sources, or at least some of them, could be telling us more about protostellar formation in extreme environments than about the very earliest phases of protostellar collapse.

A related question is, why did the standard model of singular isothermal sphere collapse work so well in Taurus? We suggest that this is simply due to initial conditions. In the absence of any complicating factors - magnetic field support, turbulent motions, etc. - thermal gas pressure provides an absolute lower limit to the forces resisting gravitational collapse, especially as cosmic ray heating seems to maintain cloud temperatures at a minimum value of $\sim 10 \mathrm{~K}$ in the absence of any other heat sources (Goldsmith 1988). Absent substantial turbulent support, or high-density flows, Taurus may only represent a limiting case of star formation.

\section{Summary}

We have described radiative transfer calculations for the spectral energy distributions of recently identified Class 0 protostars. Our models require infall rates on the order of $10^{-4} \mathrm{M}_{\odot} \mathrm{yr}^{-1}$ to account for the observed SEDs. The sub-millimeter intensity profiles of the Class 0 source VLA 1623 apparently imply a shallower density distribution than that produced by infall. But we show that material exterior to the infall region may contribute to the observed intensity profiles and thus infall models (with exterior emission) can be consistent with observations. The high infall rates in Class 0s, as required by our models and implied by a variety of observations, may be inevitable given their dense environments. Thus, we suggest that protostars in dense regions are more likely to appear as Class 0 sources while those in lower-density regions manifest Class I characteristics. The Class 0/Class I division may thus represent initial conditions more than evolutionary status, at 
least for some sources.

We are grateful to the referee, Dr. S. Terebey, for a thorough review. This work was supported by the Smithsonian Institution. 


\section{REFERENCES}

Adams, F.C., Lada, C.J., \& Shu, F.H. 1987, ApJ, 312, 788 (ALS)

Adams, F.C. \& Shu, F.H. 1986, ApJ, 308, 836

André, P., Martin-Pintado, J. Despois, D., \& Montmerle, T. 1990a, A\&A, 236, 180

André, P., Montmerle, T., Feigelson, E.D., \& Steppe, H. 1990b, A\&A, 240, 321

André, P. 1997, in Herbig-Haro Flows and the Birth of Low Mass Stars, ed. B. Reipurth \& C. Bertout (Dordrecht: Kluwer), 483

André, P., \& Montmerle, T. 1994, ApJ, 420, 837

André, P., Motte, F., \& Bacmann, A. 1999, ApJ, 513, L57

André, P., Ward-Thompson, D. \& Barsony, M. 1993, ApJ, 406, 122 (AWB)

André, P., Ward-Thompson, D. \& Barsony, M. 2000, in Protostars and Planets IV, eds. V. Mannings, A.P. Boss, \& S.S. Russell (Tucson: Univ. of Arizona Press), 59 (AWB2)

André, P., Ward-Thompson, D., \& Motte, F. 1996, A\&A, 314, 625

Ballesteros-Paredes, J. Vázquez-Semadeni, E., \& Scalo, J.M. 1999, ApJ, 515, 286

Barsony, M. 1994, in Clouds, Cores and Low Mass Stars, ed. D.P. Clemens \& R. Barvainis (Provo: Astronomical Society of the Pacific), 197

Benson, P.J. \& Myers, P.C. 1989, ApJS, 71, 89

Bontemps, S., André, P., \& Ward-Thompson, D. 1995, A\&A, 297, 98

Bontemps, S., André, P., Terebey, S., \& Cabrit, S. 1996, A\&A, 311, 858

Calvet, N., Hartmann, L., Kenyon, S.J. \& Whitney, B.A. 1994, ApJ, 434, 330

Chandler, C.J., \& Richer, J.S. 2000, ApJ, 530, 851

Chen, H., Myers, P.C., Ladd, E.F., \& Wood, D.O.S. 1995, ApJ, 445, 377

Chini, R., Krügel, E., Haslam, C.G.T., Kreysa, E., Lemke, R., Reipurth, B., Sievers, A., \& Ward-Thompson, D. 1993, A\&A, 272, L5

Draine, B.T. \& Lee, H.M. 1984, ApJ, 285, 89

Ferreira, J. \& Pelletier, G. 1995, A\&A, 295, 807

Foster, P.N. \& Chevalier, R.A. 1993, ApJ, 416, 303

Fuller, G.A. \& Myers, P.C. 1993, ApJ, 418, 273

Galli, D. \& Shu, F.H. 1993a, ApJ, 417, 220

Galli, D. \& Shu, F.H. 1993b, ApJ, 417, 243 
Goldsmith, P.F. 1988, in Molecular clouds in the Milky Way, Lecture Notes in Physics, ed. R.L. Dickman, R.L. Snell, J.S. Young (Springer-Verlag), vol. 315, 1

Hartmann, L., Boss, A., Calvet, N., \& Whitney, B. 1994, ApJ, 430, 49

Hartmann, L., Calvet, N., \& Boss, A. 1996, ApJ, 464, 387

Henriksen, R., André, P., \& Bontemps, S. 1997, A\&A, 323, 549

Hogerheijde, M.R., \& Sandell, G. 2000, ApJ, 534, 880

Hurt, R.L. \& Barsony, M. 1996, ApJL, 460, L45

Kenyon, S.J., Hartmann, L.W., Strom, K.M., \& Strom, S.E. 1990, AJ, 99, 869

Kenyon, S.J., Calvet, N. \& Hartmann, L. 1993, ApJ, 414, 676 (KCH)

Kenyon, S.J., Gomez, M., Marzke, R.O., \& Hartmann, L. 1994, AJ, 108, 251

Lada, C.J. 1987, in Star Forming Regions, ed. M. Peimbert \& J. Jugaku (Dordrecht: Reidel), 1

Lada, C.J. \& Wilking, B. A. 1984, ApJ, 287, 610

Ladd, E.F., Fuller, G.A., \& Deane, J.R. 1998, ApJ, 495, 871

Larson, R.B. 1969, MNRAS, 145, 271

Lefloch, B., Eisenhoffel, J., \& Lazareff, B. 1996, A\&A, 313, 17

Leous, J.A., Feigelson, E.D., André, P., \& Montmerle, T. 1991, ApJ, 379, 683

Li, Z.Y. \& Shu, F.H. 1996, ApJ, 472, 211

Lizano, S., \& Shu, F.H. 1989, ApJ, 342, 834

Mardones, D., Myers, P.C., Tafalla, M., Wilner, D.J., Bachiller, R., \& Garay, G. 1997, ApJ, 489,719

Motte, F., André, P., \& Neri, R. 1998, A\&A, 336, 150

Mouschovias, T.Ch. 1991, ApJ, 373, 169

O’Linger, J.C., Wolf-Chase, G., \& Barsony, M. 1997, BAAS, 191, 716

Penston, M.V. 1969, MNRAS, 144, 425

Pudritz, R.E., Wilson, C.D., Carlstrom, J.E., Lay, O.P., Hills, R.E., Ward-Thompson, D. 1996, ApJL, 470, L123

Shu, F.H. 1977, ApJ, 214, 488

Shu, F.H., Adams, F.C., \& Lizano, S. 1987, ARA\&A, 25, 23

Shu, F., Najita, J., Ostriker, E., Wilkin, F., Ruden, S., \& Lizano, S. 1994, ApJ, 429, 781 
Stone, J.M., Ostriker, E.C., \& Gammie, C.F. 1998, ApJ, 508, L99

Terebey, S., Chandler, C.J., \& André, P. 1993, ApJ, 414, 759

Terebey, S., Shu, F.H., \& Cassen, P. 1984, 286, 529 (TSC)

Ward-Thompson, D., Chini, R., Krügel, E., André, P., \& Bontemps, S. 1995, MNRAS, 274, 1219

Ward-Thompson, D., Motte, F., \& André, P. 1999, MNRAS, 305, 143

Ward-Thompson, D., Scott, P.F., Hills, R.E., \& André, P. 1994, MNRAS, 268, 276

Zhou, S., Wu, Y., Evans, N.J.,II, Fuller, G.A., \& Myers, P.C. 1989, ApJ, 346, 168 


\section{Figure Captions}

Figure 1 - (a) Spectral energy distribution of VLA 1623 using data from AWB. (The expected contribution from free-free emission at long wavelengths has been subtracted.) The solid curve is an infall model with $\dot{M} \sim 2 \times 10^{-4} M_{\odot}$, and the dotted curve is a model with $\dot{M} \sim 10^{-5} M_{\odot}$. (b) Spectral energy distribution of HH24MMS using data from Ward-Thompson et al. (1995). The solid curve is an infall model with $\dot{M} \sim 3 \times 10^{-4} M_{\odot}$.

Figure 2 - Comparison of the radial intensity profiles of VLA 1623 at $800 \mu \mathrm{m}, 450 \mu \mathrm{m}$, and $350 \mu \mathrm{m}$ with the basic infall model in which $\rho \propto r^{-3 / 2}$ (dotted line), the infall model where $T_{\min }=20 \mathrm{~K}$ (dashed line), and the composite model described in Section 3 which includes a contribution from regions exterior to the infalling core (solid line). The model output was convolved with the appropriate beam size at each wavelength and scaled to a distance of $160 \mathrm{pc}(13.5 "=2160 \mathrm{AU}, 7.5 "=1200 \mathrm{AU}$, and $6.3 "=1000 \mathrm{AU}$, respectively $)$.

Figure 3 - Comparison of the spectral energy distribution of VLA 1623 to the best-fit composite model (solid line). The dotted line represents emission of the infall region while the dashed line shows the contribution of the exterior "slab." 

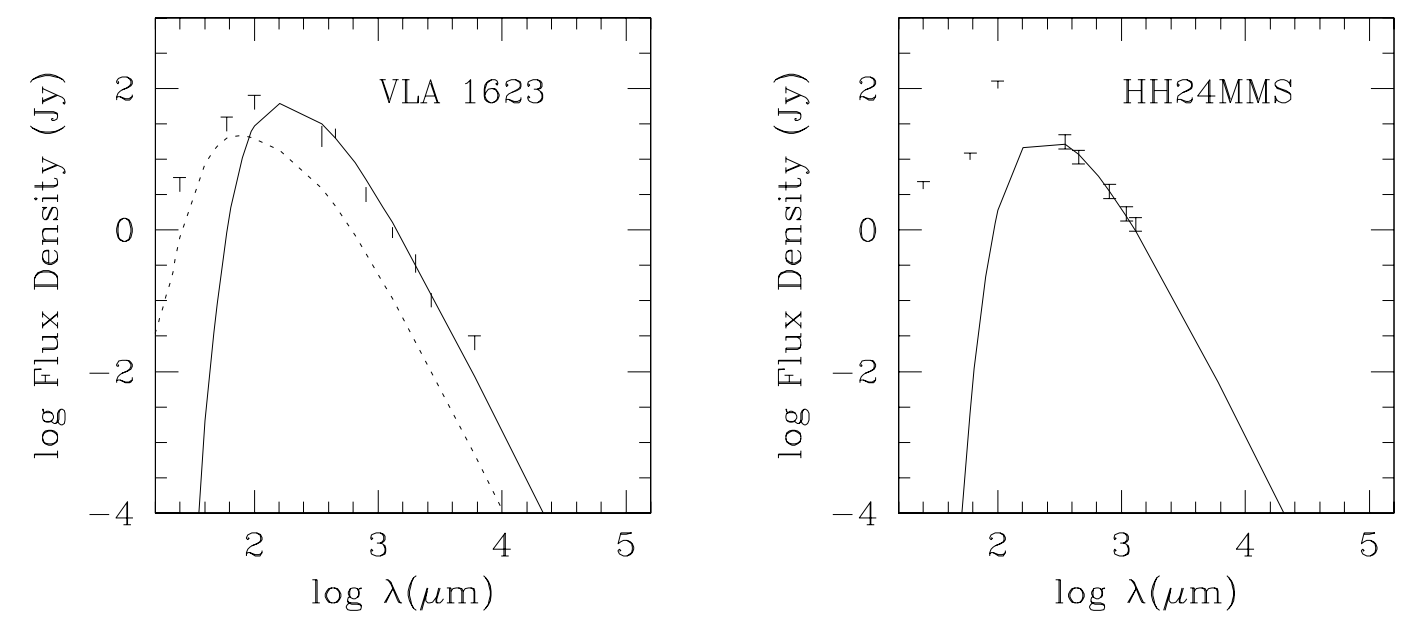

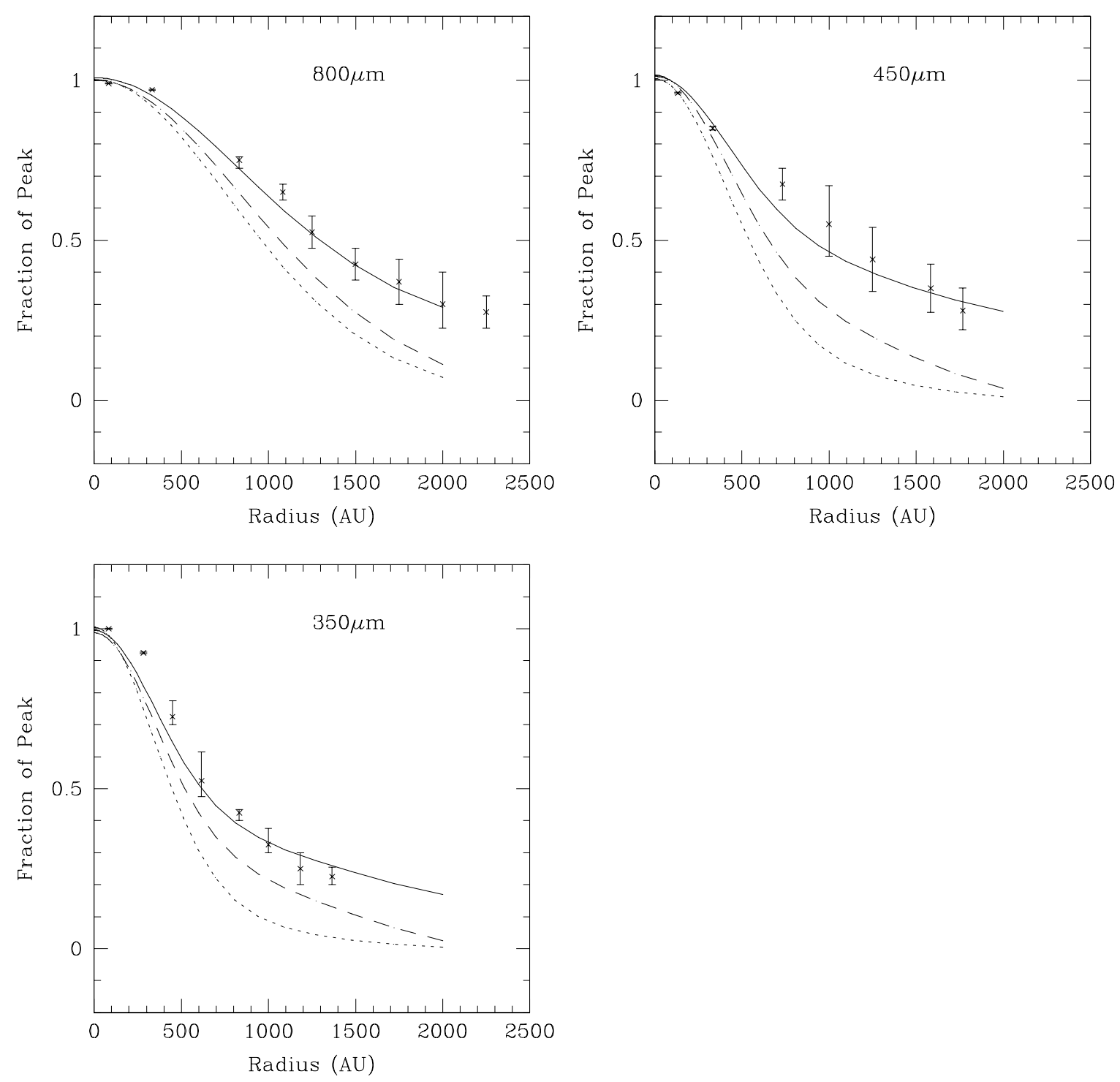


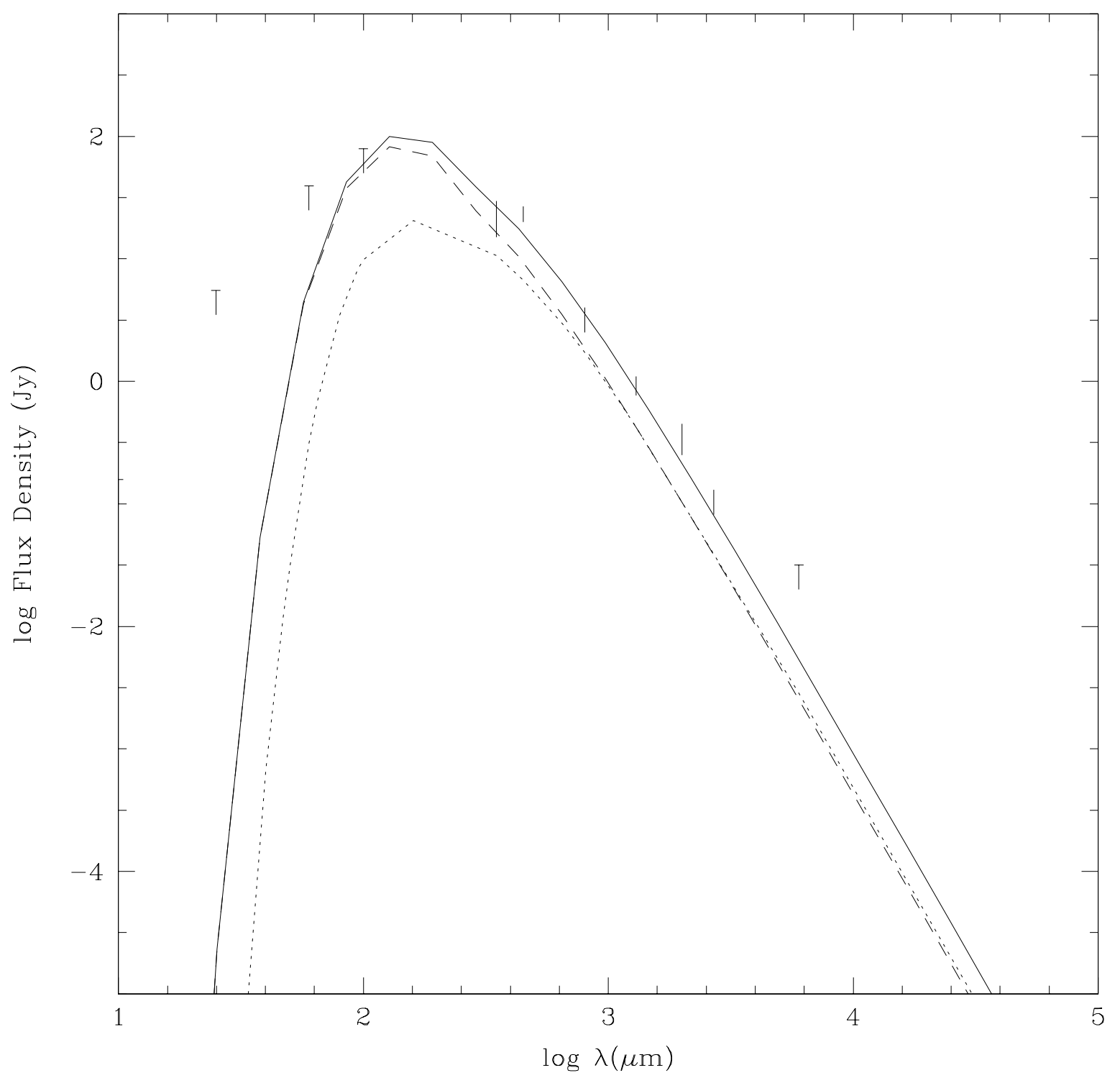

